City University of New York (CUNY) CUNY Academic Works

1984

\title{
(Super)conformal Symmetry Breaking
}

Andrew Strominger

The Institute for Advanced Study

V. Parameswaran Nair

City College of New York

\section{How does access to this work benefit you? Let us know!}

More information about this work at: https://academicworks.cuny.edu/cc_pubs/466

Discover additional works at: https://academicworks.cuny.edu

This work is made publicly available by the City University of New York (CUNY).

Contact: AcademicWorks@cuny.edu 


\title{
(Super)conformal symmetry breaking
}

\author{
Andrew Strominger and V. P. Nair \\ The Institute for Advanced Study, Princeton, New Jersey 08540
}

(Received 3 August 1984)

\begin{abstract}
We argue that the only consistent way to break conformal invariance in a quantum theory of gravity is spontaneously in a finite locally conformal supersymmetric theory. This generically occurs whenever scalar fields are present. Covariant surface-integral expressions for the supercharge and four-momentum in terms of asymptotically constant spinor fields are derived. These are used to discuss stability and other aspects of the theory.
\end{abstract}

\section{INTRODUCTION}

Higher-derivative theories of gravity have received much attention as candidates for a fundamental quantum theory of gravity. ${ }^{1}$ Among the general class of renormalizable fourth-order theories with matter, the conformally supersymmetric ones stand out as particularly interesting members. One reason for interest in conformally invariant theories has been the decrease in the number of allowed coupling constants and the consequent increase in predictive power. $^{2}$ Another reason is that, unlike the general fourth-order theory, classical solutions of conformally invariant gravity always have zero energy, ${ }^{3}$ which could be relevant to the stability/unitarity of the quantum theory. ${ }^{3,4}$ Many efforts have been made, dating back to Weyl, ${ }^{5}$ to incorporate conformal invariance into the fundamental laws of physics. ${ }^{6,7}$ However (as we shall argue) conformal invariance plus renormalizability implies supersymmetry and we are thus led to study conformal supergravity.

In this paper we investigate several aspects of conformally supersymmetric theories. In Sec. II we give an argument that the only quantum gravitational field theories that consistently incorporate conformal invariance are finite conformally supersymmetric ones. Since superconformal invariance clearly is not a manifest symmetry of the real world, it must somehow be broken. Mechanisms for spontaneous breakdown of superconformal invariance are illustrated in Sec. III. It is shown that symmetry breaking generically occurs whenever there are scalars present and that the resultant cosmological constant will be zero if there are flat directions in the potential. The symmetry breaking is best understood in terms of the surface-integral expressions for the symmetry generators. In Sec. IV we derive the covariant expressions for the total supercharge and four-momentum. This gives us the analog of Witten's expression ${ }^{8}$ for conformal gravity. The difficulties with ghosts are briefly discussed.

\section{CONFORMAL INVARIANCE IN QUANTUM GRAVITY}

A conformal transformation is a transformation under which the metric is multiplied by an arbitrary scalar function:

$$
g_{a b}(x) \rightarrow \Omega^{2}(x) g_{a b}(x) .
$$

Such a transformation generally cannot be obtained by a change of coordinates, and the conformally related metrics are regarded, in general relativity, as physically inequivalent. However, in a theory described by a conformally invariant action, the evolution equations will only determine the metric up to a local conformal factor. This means that only conformally invariant quantities can have physical significance. In particular, the distance between two points is not an invariant concept, although their causal relation is. Invariant local information about the geometry is summarized by the Weyl tensor

$C_{a b c}{ }^{d}=R_{a b c}{ }^{d}+g_{c[b} R_{a]}{ }^{d}+g_{[a}^{d} R_{b] c}+\frac{1}{3} R g_{c[a} g_{b]}{ }^{d}$,

where $\left[\nabla_{a}, \nabla_{b}\right] \epsilon_{c}=R_{a b c}{ }^{d} \epsilon_{d}$ and $R_{a b}=R_{a d b}{ }^{d}$.

In nongravitational theories such as Yang-Mills theory, conformal invariance is explicitly broken at the quantum level via dimensional transmutation. Because of the unavoidable introduction of a length scale in the renormalization process, the quantum effective action is not conformally invariant. Attempts have been made to break, in the same fashion, the conformal invariance of the gravitational action

$$
S_{c}=-\frac{1}{\alpha^{2}} \int d^{4} x \sqrt{-g} C_{a b c d} C^{a b c d} .
$$

However, this does not seem to be a sensible procedure (at least within the context of standard regularization and renormalization prescriptions. There is always the possibility that a nonstandard treatment could yield a consistent theory). ${ }^{6}$ In order to renormalize (3), all counterterms of dimension four or less- $C_{a b c d} C^{a b c d},{ }^{*} R_{a b c d}^{*} R^{a b c d}, R^{2}, R$, and $\Lambda$-will eventually be needed. ${ }^{9}$ One might attempt to argue that the nonconformally invariant counterterms could be absorbed by renormalization of the conformal gauge-fixing terms. However, because of the trace anomaly, even expectation values of "conformally invariant" operators will depend both on the coefficients of these counterterms and the conformal gauge-fixing terms. To see this, consider the formally conformal invariant expectation value

$$
\langle O\rangle=\left\langle\sqrt{-g} C_{a b c d} C^{a b c d}(x)\right\rangle .
$$


If we now perform an infinitesimal conformal transformation at $y \neq x$, we find

$$
\begin{aligned}
\delta_{c}\langle O\rangle= & \frac{\beta(\alpha)}{\alpha^{2}}\left\langle\sqrt{-g} C_{a b c d} C^{a b c d}(y) \sqrt{-g} C_{a b c d} C^{a b c d}(x)\right\rangle \\
& +\left\langle T_{\mathrm{NI}}(y) \sqrt{-g} C_{a b c d} C^{a b c d}(x)\right\rangle
\end{aligned}
$$

where $T_{\mathrm{NI}}$ is that part of the trace anomaly which is not invariant under conformal transformations. Since the two terms in (5) have different conformal transformation properties, they cannot cancel. The first term cannot vanish as it has a positive spectral representation [assuming we quantize with a positive metric. However, even with negative-metric quantization there is no reason (5) should vanish.] $\langle O\rangle$ is therefore not conformally invariant. [Of course if conformal invariance is spontaneously broken, one does not expect invariance under global conformal transformations. However, in (5) we have subjected $\langle O\rangle$ to a local conformal transformation.] All expectation values will thus depend on the coefficients of the noninvariant counterterms and they must be regarded as physical quantities. The theory thus becomes equivalent to the general fourth-order theory and retains no relic of conformal invariance. ${ }^{10}$ Put another way, quantum divergences lead to an anomalous divergence in the conformal current and theories with anomalies in their gauge currents are in no sense gauge invariant at the quantum level.

To avoid this difficulty, one follows the standard procedure for dealing with anomalous gauge currents: the matter representations are required to be such that the net conformal anomaly vanishes due to cancellations. ${ }^{10}$ This provides a very severe constraint, viz., the theory must be finite. The existence of a class of finite conformal supergravity theories has been argued by Howe et al. ${ }^{11}$ This class of finite theories is analogous to the class of finite super Yang-Mills theories, and the argument for finiteness proceeds along similar lines. Briefly, background superfield formulations of some $N$-extended conformal supergravity theories coupled to $N$-extended conformal supermatter have been found. Within such a formulation, dimensional arguments can be used (for $N>1$ ) to show that there are no divergences except at one loop where extra ghosts couple to the background superfield. Finiteness then follows if the matter representations can be chosen so that the one-loop divergences cancel. In principle this should be possible, but a classification of finite theories has not yet appeared in the literature.

\section{BREAKING SUPERCONFORMAL SYMMETRY}

Once local conformal invariance of the full quantum theory has been ensured by finiteness, we are still faced with the task of breaking the global conformal invariance. This can occur ${ }^{12}$ spontaneously, without inducing a cosmological constant, whenever there is a scalar field with flat directions in its potential. Such potentials are in fact quite common in supersymmetric models, e.g., $N=4$ Yang-Mills. In many cases nonrenormalization theorems maintain the flat directions to all orders in perturbation theory. As an explicit example, consider $N=2$ conformal supergravity coupled to an $N=2$ vector multiplet. (This model was discovered and used by de Wit and collaborators as a mathematical tool for deriving $N=2$ Poincaré supergravity actions. ${ }^{13}$ ) (We do not know if this is part of some finite theory.) The relevant part of the action is

$$
S_{c}+\int d^{4} x \sqrt{-g}\left(\frac{1}{2} D_{a} \varphi^{*} D^{a} \varphi-\frac{1}{12} R \varphi^{*} \varphi+\cdots\right),
$$

where $D_{a}=\nabla_{a}-i A_{a}, A_{a}$ is the chiral U(1) gauge field and $\varphi$ is the complex scalar field of the vector multiplet. It can be arranged (e.g., by coupling an additional scalar or nonlinear multiplet ${ }^{13}$ ) so that the potential for $\varphi$ vanishes. We then choose $\varphi=\varphi_{0}$ as a homogeneous, isotropic classical solution around which to expand the quantum theory.

One might ask if this classical solution minimizes the energy with appropriate boundary conditions. As we will show in Sec. IV, the conserved energy of this theory is given by a surface integral at spatial infinity

$$
E=\frac{8}{\alpha^{2}} \int d^{2} S_{j} \nabla_{i} C^{0 i j}{ }_{0}^{j}+\frac{4 \pi}{3} \varphi_{0}{ }^{2} E_{\mathrm{ADM}}-\frac{1}{6} \varphi_{0} \int d^{2} S_{j} \varphi_{, j},
$$

where

$$
E_{\mathrm{ADM}}=\frac{1}{16 \pi} \int d^{2} S_{j}\left(g_{i j, j}-g_{j j, i}\right)
$$

is the standard Arnowitt-Deser-Misner (ADM) energy of general relativity. We have explicitly allowed for the possibility that $\varphi$ tends asymptotically to the constant $\varphi_{0}$. The first term on the right-hand side of (7) is in fact always zero because the constraint equations force $D_{j} C_{0}{ }^{i 0 j}$ to fall off faster than $r^{-2}$ at spatial infinity. This implies that when $\varphi_{0}=0$ the total energy is always zero. When $\varphi_{0} \neq 0$, it is easy to see that there are many solutions with positive energy (consider a metric that satisfies the vacuum Einstein equation and set $\varphi=\varphi_{0}$ everywhere). An analysis of the linearized equations around flat space reveals negative-energy modes (whether or not $\varphi=0$ ). However solutions of the linearized equations in a fourth-order theory do not in general correspond to the limit of a oneparameter family of exact solutions (this is known as linearization instability), so such an analysis could be misleading [and is when $\varphi=0$ (Refs. 3 and 14)]. However, although no explicit examples are known, arguments have recently been given indicating that exact negativeenergy solutions do in fact exist. ${ }^{14}$ In any case, we are certainly unable to argue that our $\varphi=\varphi_{0}$, or any other solution, indeed minimizes the classical energy. This is of course the central difficulty with higher-derivative gravity theories, and is the classical origin of the quantum unitarity problems. Further discussion of this point may be found in Ref. 15. For the rest of this paper we will ignore all questions of stability.

A nonzero vacuum expectation value for $\varphi$ breaks both chiral and conformal invariance. The $N=2$ conformal supersymmetry is broken down to $N=2$ Poincare supersymmetry. However, there are no Goldstone bosons due to the Higgs phenomenon. One may simultaneously fix unitary chiral and conformal gauges by requiring $\varphi=\varphi_{0}$. The degree of freedom in the phase of $\varphi$ is absorbed by $A_{a}$, which acquires a mass. The rest of $\varphi$ is absorbed by the conformal part of the metric. Before symmetry breaking, a linearized analysis reveals that the metric con- 
tains two massless spin-two and one massless spin-one degrees of freedom. ${ }^{16}$ (Of course since this theory is linearization unstable, ${ }^{3}$ it is somewhat inappropriate to discuss linearized modes. It would be more precise, but more cumbersome, to rephrase these statements in terms of Cauchy data.) This large number of degrees of freedom is due to the presence of higher derivatives. The linearized action does not involve the trace of the linearized fluctuations. When $\varphi=\varphi_{0}$, however, the trace $h$ enters the linearized quadratic action in the form

$$
\frac{1}{128} \varphi_{0}^{2} \nabla_{a} h \nabla^{a} h
$$

and therefore propagates. A fuller analysis reveals that it combines with the massless spin-one and one of the massless spin-two particles to form a massive spin-two ghost or tachyon. This ghost (tachyon) sits at the top of an entire ghost (tachyon) supermultiplet.

The massless linearized modes are of particular interest because they are relevant to low-energy physics and because, unlike the massive case, it can be easily shown that massless solutions to the linearized equations are also linearizations of exact solutions. This follows from the fact that exact solutions to the Einstein equations are also exact solutions to the Weyl equations. In particular there are exact plane-wave solutions that, when linearized, correspond to the familiar graviton. The other massless excitations can then be obtained by supersymmetry transformations. The massless spectrum thus consists of the standard $N=2$ supergravity multiplet and is ghost free.

We now consider the case where there are no flat directions in the potential. The relevant part of the action is

$$
\begin{array}{r}
S=S_{c}+\int d^{4} x \sqrt{-g}\left[\frac{1}{2} D_{a} \varphi^{*} D^{a} \varphi-\frac{1}{12} \varphi^{*} \varphi R\right. \\
\left.-g\left(\varphi^{*} \varphi\right)^{2}+\cdots\right] .
\end{array}
$$

One might at first think that conformal invariance is not spontaneously broken with this action. Note, however, that there is a homogeneous, isotropic solution

$$
\varphi=\varphi_{0}, \quad R^{c d}\left(g_{a b}\right)=-6 g \varphi_{0}^{2} g^{0 c d}
$$

$g^{0 c d}$ is the anti-de Sitter metric. In the gauge $\varphi=\varphi_{0}$, the action becomes

$$
S=S_{c}-\frac{\varphi_{0}{ }^{2}}{12} R-g \varphi_{0}^{4}
$$

from which we see that conformal symmetry breaking has produced a negative, Planck-sized cosmological constant. We expect that analysis of the appropriate surface integrals will indicate that the symmetry has been broken down to $N=2$ de Sitter supersymmetry. It thus appears that conformal invariance will generically be spontaneously broken as long as there are scalars present.

We stress that the breakdown of conformal invariance is a dynamical issue. The statement has appeared in the literature that conformal theories with scalars are "gauge equivalent" to nonconformal theories and that this can be seen by going to the gauge $\varphi^{2}=$ constant. This is incorrect because if $\varphi=0$ there is no gauge transformation that sets it to a constant. (This does not prevent one from setting $\varphi=$ constant as a mathematical tool for obtaining Poincaré supergravity theories from conformal supergravity theories. This technique has in fact been used quite fruitfully.) At the classical level, the broken and unbroken phases of the theory are radically different: one has only zero energy, the other does not. An analogous situation occurs in Yang-Mills-Higgs theories. If there is a symmetry-breaking potential, the $\Phi_{a}=$ constant gauge choice is appropriate, otherwise it is not.

We have also found that with more complicated actions it can be arranged so that supersymmetry is spontaneously broken as well. For the potentials we considered, the effective cosmological constant is not zero unless certain couplings are fine tuned.

\section{ENERGY AND SUPERCHARGE IN CONFORMAL SUPERGRAVITY}

The total four-momentum and supercharge of an isolated system are, in a locally supersymmetric theory, globally conserved quantities derived from a local symmetry. It follows from this that they are expressible as surface integrals. In this section we will derive these surface integrals and see that supersymmetry relates them.

The action for $N=1$ conformal supergravity is, in two-component notation,

$$
\begin{aligned}
& S=\int\left\{\left(\nabla_{C}{ }^{\dot{X}} \nabla_{B} \dot{Y}-\Phi_{C B}{ }^{\dot{X} \dot{Y}}\right) \psi_{A(\dot{X} \dot{Y})} \nabla_{\dot{Z}}{ }^{(A} \psi^{B C) \dot{Z}}\right. \\
& -\Psi^{A B C D}\left[\psi_{A(\dot{X} \dot{Y})} \nabla_{B} \dot{X}_{C D} \psi_{C}+2\left(\nabla_{B} \dot{X}_{A(\dot{X} \dot{Y})}\right) \psi_{C D} \dot{Y}\right] \\
& \left.-\frac{1}{12} \varphi_{0}^{2} \psi_{A \dot{X} \dot{Y}}\left(\nabla^{A} \dot{Y}^{B} \psi_{B}^{B}-\nabla^{B \dot{X}} \psi_{B}^{A \dot{Y}_{B}}\right)+\text { H.c. }+L_{m}\right\} \text {, }
\end{aligned}
$$

where $\Phi_{A B \dot{A} \dot{B}}=-\frac{1}{2} R_{a b}$ and

$$
C_{a b c d}=\Psi_{A B C D} \epsilon_{\dot{A} \dot{B}} \epsilon_{\dot{C} \dot{D}}+\Psi_{\dot{A} \dot{B} \dot{C} \dot{D}} \epsilon_{A B} \epsilon_{C D},
$$

where $L_{m}$ represents the matter and gravity action and terms of higher order in $\psi$. In the $N=2$ action $\psi$ has an index that runs one to two. It is worth noting that the use of two-component notation dramatically simplifies the action and the subsequent calculation. The Majorana condition on $\psi$ implies

$$
\left(\psi_{M \dot{M} \dot{A}}\right)^{*}=\psi_{\dot{M} M A}
$$

The invariance of the $\varphi_{0}=0$ part of the action under $S$ supersymmetry

$$
\delta_{S} \psi_{M \dot{M} \dot{A}}=\epsilon_{\dot{M} \dot{A}} \eta_{M}
$$

is manifest because (12) involves only the symmetric part of $\psi$. The invariance under $Q$ supersymmetry $\left(\delta_{Q} \psi_{\dot{M} M \dot{A}}=\nabla_{\dot{M} M} \alpha_{\dot{A}}\right)$ leads, in a Hamiltonian formulation, to constraints on the initial data on a spacelike surface $\Sigma$. These are simply the time component of the $\psi$ equation of motion

$$
\begin{aligned}
C^{A \dot{X} \dot{Y}}= & \left.\nabla_{B}{ }^{\dot{Y}} \nabla_{C}{ }^{\dot{X}} \nabla_{\dot{Z}}{ }^{(A} \psi^{B C) \dot{Z}}+\nabla_{\dot{Z}}{ }^{A} \nabla_{X}{ }^{(\dot{X}} \nabla_{Y} \dot{Y} \psi^{\dot{Z}}\right)(X Y) \\
& +\frac{1}{12} \varphi_{0}{ }^{2}\left(\nabla^{B \dot{X}_{Y}} \psi^{A \dot{Y}}{ }_{B}-\nabla^{A} \dot{Y}_{\left.\psi^{B} \dot{X}_{B}\right)}\right. \\
& -\varphi\left(\epsilon^{\dot{X} \dot{Y}} \nabla^{A \dot{A}} \lambda_{\dot{A}}-2 \nabla^{A\left(\dot{X}_{\lambda} \dot{Y}\right)}\right)-J^{A \dot{X} \dot{Y}}
\end{aligned}
$$


$J$ represents source terms that fall off faster than $r^{-3}$ at spatial infinity and $\lambda$ is the superpartner of $\varphi$. $Q$ supersymmetry transformations that go to zero at infinity are generated, after a canonical decomposition, ${ }^{17,18}$ by the integral of the constraint over the surface $\Sigma$,

$$
\int d^{3} \Sigma_{A \dot{A}}\left(C^{A \dot{A} \dot{X}} \alpha_{\dot{X}}+\text { c.c. }\right) \text {. }
$$

If $\alpha$ goes to a constant at infinity, (16) does not generate anything because it involves spatial derivatives of the canonical variables. This can be remedied by the addition of appropriate surface terms. These surface terms will be sufficient to define the generators for $\alpha$ approaching a constant spinor (plus order- $1 / r$ terms) at spatial infinity and appropriate asymptotic falloff rates for the canonical variables. When the constraint equations for both coordinate invariance and supersymmetry are satisfied the supercharge becomes

$$
\begin{aligned}
Q(\alpha)=\int d^{2} S_{A \dot{A} B \dot{B}}\left[\alpha_{\dot{X}}\right. & \epsilon^{\dot{X} \dot{B}} \nabla_{C}^{\dot{A}} \nabla_{\dot{Y}}{ }^{(A A} \psi^{B C) \dot{Y}} \\
& +\epsilon^{A B} \nabla_{X}^{(\dot{A}} \nabla_{Y}{ }^{\dot{B}} \psi^{\dot{X})(X Y)} \\
& +\frac{1}{6} \varphi^{2} \epsilon^{\dot{A} \dot{B}} \psi^{\dot{X}(A B)} \\
& \left.\left.+\varphi \epsilon^{A B} \epsilon^{\dot{X}(\dot{A}} \lambda^{\dot{B})}\right)+ \text { c.c. }\right]
\end{aligned}
$$

This expression is gauge independent, but it will only function as a generator after an appropriate gauge choice and construction of the corresponding Dirac bracket.

The commutator of two supersymmetry transformations involves a translation. As has been explained elsewhere, ${ }^{19}$ this enables us to relate the total four-momentum to the supersymmetry variation of the supercharge

$$
\begin{aligned}
P_{A \dot{A}} \alpha^{A} \alpha^{\dot{A}}=\int d^{2} S_{A \dot{A} B \dot{B}}\left[\alpha_{\dot{X}}\right. & -\epsilon^{\dot{X} \dot{B}} \nabla_{C}{ }^{\dot{A}} \nabla^{\dot{Y}}\left(A \nabla_{\dot{Y}}{ }^{B} \alpha^{C)}\right. \\
& \left.+\epsilon^{A B} \nabla_{X}{ }^{(\dot{A}} \nabla_{Y}^{\dot{B}} \nabla^{\dot{X}}\right)\left(X_{\alpha} Y\right) \\
& \left.+\frac{1}{6} \varphi^{2} \epsilon^{\dot{A} \dot{B}} \nabla^{\dot{X}(A} \alpha^{B)}\right) \\
& \left.+\alpha_{C} \varphi\left(\epsilon^{A B} \epsilon^{\dot{X}(\dot{A}} \nabla^{\dot{B}) C} \varphi\right)+\text { c.c. }\right]
\end{aligned}
$$

This is the analog of Witten's expression for conformal gravity. Although in Poincaré supergravity an expression like (18) leads to a proof of positivity of energy, the same is not true in this case. ${ }^{20}$

\section{ACKNOWLEDGMENT}

This work was supported by the Department of Energy under Grant No. DOE-AC02-76ER02220.
${ }^{1}$ For reviews see the articles by D. G. Boulware, A. Strominger, and E. Tomboulis, in Quantum Theory of Gravity, edited by S. Christensen (Adam Hilger, Bristol, 1984).

2S. Adler, Rev. Mod. Phys. 54, 729 (1982); B. Hasslacher and E. Mottola, Phys. Lett. 95B, 237 (1980); A. Zee, ibid. 109B, 183 (1983).

${ }^{3}$ D. Boulware, G. Horowitz, and A. Strominger, Phys. Rev. Lett. 50, 1726 (1983).

${ }^{4}$ M. Kaku, Nucl. Phys. B203, 285 (1982); Phys. Rev. D 27, 2819 (1983).

${ }^{5}$ H. Weyl, in Space, Time, Matter (Dover, New York, 1922).

${ }^{6}$ L. Smolin, Phys. Lett. 93B, 95 (1980); Nucl. Phys. B160, 253 (1979); I. Antoniadis and N. Tsamis, Report No. SLACPUB-3296, 1984 (unpublished).

${ }^{7}$ M. Kaku, P. Townsend, and P. van Nieuwenhuizen, Phys. Rev. D 17, 3179 (1978); Phys. Rev. Lett. 39, 1109 (1977).

${ }^{8}$ E. Witten, Commun. Math. Phys. 80, 381 (1981).

${ }^{9}$ F. David and A. Strominger, Phys. Lett. 143B,125 (1984).

${ }^{10}$ Similar conclusions have been reached by $\mathrm{E}$. Fradkin and $\mathrm{A}$ Tseytlin, Lebedev Institute Report No. 185, Moscow, 1983 (unpublished); Phys. Lett. 134B, 187 (1984).

${ }^{11}$ P. S. Howe, K. S. Stelle, and P. K. Townsend, Nucl. Phys. B236, 125 (1984).
${ }^{12}$ Y. Fujii, Phys. Rev. D 9, 874 (1974). See also, in the context of supersymmetry, M. Kaku and P. K. Townsend, Phys. Lett. 76B, 54 (1978).

${ }^{13}$ There is an extensive literature on extended conformal supergravity. See B. de Wit, J. van Holten, and A. van Proeyen, Nucl. Phys. B184, 77 (1981), and references therein.

${ }^{14} \mathrm{R}$. Woodard (private communication).

${ }^{15}$ E. Tomboulis, Phys. Rev. Lett. 52, 1173 (1984).

${ }^{16}$ S. C. Lee and P. van Nieuwenhuizen, Phys. Rev. D 26, 934 (1982).

${ }^{17}$ A. Hanson, T. Regge, and C. Teitelboim, Constrained Hamiltonian Systems (Academia Nazionale de Lincei, Rome, 1976).

${ }^{18}$ For a canonical analysis of higher-derivative Rarita-Schwinger actions, see D. Boulware and S. Deser, Phys. Rev. D 30, 707 (1984).

${ }^{19}$ G. T. Horowitz and A. Strominger, Phys. Rev. D 27, 2793 (1983); S. Deser, ibid. 27, 2805 (1983); C. M. Hull, Commun. Math. Phys. 90, 545 (1983); C. Teitelboim, Phys. Rev. D 29, 2763 (1984).

${ }^{20}$ Further discussion of this point in the context of supersymmetry can be found in Ref. 18 and D. G. Boulware, S. Deser, and K. S. Stelle (unpublished). 